\title{
A Brief Survey of String/Brane Cosmology
}

\section{Sudhakar Panda*}

Harish-Chandra Research Institute, Allahabad

E-mail:panda@mri.ernet.in

In this short review, we recapitulate some features of the cosmological standard model, followed by a survey of various ideas in cosmological applications of string theory without detailed analysis of any particular model. This includes ideas from the pre-D-brane epoch as well as inflationary scenarios inspired by D-brane physics.

From String to $\mathrm{LHC}$

January 2-10, 2007

Goa, India

${ }^{*}$ Speaker. 


\section{Introduction}

In recent times it has been a general perception that string theory and cosmology have to complement each other in some ways. On one hand, cosmology needs an underlying fundamental theory of gravity coupled to matter which can address its basic questions such as initial singularity, origin of inflation or any other alternative to inflation which can solve problems like the horizon and flatness. It should also account for the origin of density perturbations in cosmic microwave background (CMB) among many other issues. Infact the success of inflation, as a model to solve most of the above issues, has made us so comfortable that we tend to overlook the fact that inflation is only a scenario in search of an underlying theory. The need of the moment is a firm derivation of inflation or its alternatives from a more fundamental theory such as string theory or M-theory and we will discuss some progress in this direction. On the other hand, string theory, as it is developed and understood till now, is far from being subject to any stringent test. The hope at present is the low energy brane-world phenomenology with supersymmetry could provide hints to its high energy origin or some of the low string-scale physics could be probed at LHC. If such a hope turns out to be a wishful thinking, the only arena we will be left with, possibly, is cosmology. This could turn out to be the avenue to probe string theory and the proposed experiments such as MAP and PLANCK can shed some light on the future direction for string theory. Thus what we are advocating here is that the study of cosmological relevance of string theory can help to have a proper understanding of string theory itself in the investigation of various issues like the cosmological constant problem, identification of initial conditons, initial singularity, realizing a de Sitter background, cosmological scaling solutions etc. In the next section we will discuss some salient features of cosmological standard model and inflation.

\section{Cosmological standard model and Inflation}

The standard cosmological model begins with the Einstein's equations in the presence of matter. One assumes the four dimensional space-time to be homogeneous (same at every point) and isotropic (same in every direction). Of course these are only approximate, but they become increasing good approximations on larger length scales. These requirements determines the metric upto an arbitrary function $a(t)$ and a discrete parameter $k$. While $a(t)$, called the scale factor, describes the relative size of spacelike hypersurfaces at different times, $k$ takes values $-1,0$ and 1 corresponding to the universe being open (negatively curved hypersurfaces), flat (flat hypersurfaces) and closed (positively curved hypersurfaces) respectively. The Friedmann-Robertson-Walker metric which describes the evolution of the universe is written as:

$$
d s^{2}=-d t^{2}+a^{2}(t)\left[\frac{d r^{2}}{\left(1-K R^{2}\right)}+r^{2}\left(d \theta^{2}+\sin ^{2} \theta d \phi^{2}\right)\right]
$$

The scale factor $a(t)$ is determined by solving the Einstein equation:

$$
G_{\mu v} \equiv R_{\mu v}-\frac{1}{2} g_{\mu v} R=-8 \pi G T_{\mu \nu}
$$

where the Newton's constant $G$ is given by $G=1 / 8 \pi M_{p l}^{2}$ in the unit of $h=c=1$ and the stressenergy tensor $T_{\mu \nu}$ is taken corresponding to a perfect fluid i.e.

$$
T_{\mu v} d x^{\mu} d x^{v}=\rho(t) d t^{2}+p(t) g_{i j} d x^{i} d x^{j}
$$


where $\rho(t)$ is called the energy density and $p(t)$ is called the pressure. The weak energy conditon requires that the energy density is non-negative. Eliminating the coordinate $t$ one finds that the pressure and the energy density satisfy an equation of state $p=\omega \rho$. The parameter $\omega$ takes a constant value corresponding to the type of matter that dominates the stress-energy tensor. For example, in the case of a flat universe, $\omega=-1,0$ and $1 / 3$ corresponding to vacuum with a cosmological constant $(\Lambda)$, matter dominated and radiation dominated universe respectively with respective energy density $\rho \sim \frac{\Lambda}{8 \pi G}, \rho \sim a^{-3}$ and $\rho \sim a^{-4}$. For these values of $\omega$ the corresponding scale factors are found to be $a(t) \sim e^{\sqrt{\Lambda / 3} t}, t^{2 / 3}$ and $t^{1 / 2}$ respectively. For the FRW metric given as above, Einsteins equations take the following form:

$$
\begin{aligned}
H^{2} & =\frac{8 \pi G}{3} \rho-\frac{k}{a^{2}} \\
\frac{\ddot{a}}{a} & =-\frac{4 \pi G}{3}(\rho+3 p)
\end{aligned}
$$

where $H=\frac{a}{a}$ is the Hubble function. Throughout our discussion the overdot is meant for derivative with respect to the cosmic time. While the former equation is called Friedman equation the latter is known as the Raychaudhuri equation. Together they lead to the so called "First Law" which can be expressed as

$$
\dot{\rho}=-3 H(p+\rho) .
$$

This equation expresses the fact that the energy in a co-moving volume $a^{3} \rho$ is not conserved during the expansion of the universe. Infact if we assume that the matter is in local thermodynamic equilibrium and has an adiabatic passage through a succession of equilibrium states whose equilibrium pressure is exactly $p$, then one can deduce that the entropy in a comoving volume is conserved. However, the matter is never in complete equilibrium and it always tends to the equilibrium configuration of constant entropy at a lower and lower energy (temperature). We can solve this equation making use of the equation of state to obtain $\rho=a^{-3(1+\omega)}$. This gives the behaviour of $\rho$ with respect to the scale factor as described eariler for different kinds of matter. It is worth noting that if the universe is expanding then the density of radiation falls of with one more power of the scale factor than pressure free matter, which will ultimately dominate. Similarly, the solution of the Friedmann's equation yields the behaviour of the scale factor with respect to time as it has been discussed above for the case of the flat universe. The terms in the previous equation have a simple interpretation; the term $3 H \rho$ is the reduction in energy density due to the increase in volume and the term $3 \mathrm{H}$ is the reduction in energy caused by the the thermodynamic work done by the pressure when the expansion occurs. Before we proceed further with this model, few remarks are in order. The basic assumption that the universe is spatially homogeneous and isotropic has obsevational support coming from high degree of isotropy of the cosmic microwave background radiation (CMB) and the large scale distribution of galaxies. This assumption can be put in the mathematical language by stating that locally the metric admits a six dimensional isometry group acting on three dimensional spacelike orbits $\hat{G} / S O(3)$ with $\hat{G}=S O(4), E(3)$ or $S O(3,1)$. These three possibilities correspond to the above mentioned values of $k$ equal to 1,0 and -1 respectively. Thus locally, the orbits (spatial cross sections) inherit the geometry of $S^{3}, E^{3}$ or $H^{3}$ respectively. However, globally one can make identifications on the orbits under the action of a discrete subgroup $\Gamma \subset \hat{G}$ so that the orbits $\Gamma \hat{G} / S O(3)$ become compact for $k=0,1$. Thus the names "closed" and "open" donot 
have correct meaning in a global sense though it is expected that future observations by MAP can tell us the extent to which these possibilities are actually true. For the FRW spacetime, in general the Lorentz group is spontaneously broken down to its orthogonal subgroup. In this sense, the cosmological background acts as a kind of aether which is translation invariant. But if $k \neq 0$, the translations do not commute. However, for particular scale factors the metric does admit additional isometries rendering it homogeneous in space as well as in time. Thus the metric becomes locally maximal symmetric and hence has constant curvature which implies that the metric is also Einsteinian. The following examples are interesting illustrations:

(I) de Sitter spacetime $d S_{4} \equiv S O(4,1) / S O(3,1)$ which is obtained if $k=1$ and $a(t)=\cosh (H t)$; $k=0$ and $a(t)=\exp (H t)$ or $k=-1$ and $a(t)=\sinh (H t)$ with $H^{2}=\Lambda / 3$.

(II) Minkowski spacetime $E^{3,1}=E(3,1) / S O(3,1)$ which is obtained if $k=0$ and constant $a(t)$ or $k=-1$ and $a(t)=t$.

(III) Anti-de Sitter spacetime $A d S_{4} \equiv S O(3,2) / S O(3,1)$ which is obtained if $k=-1$ and $a(t)=$ $\sin (H t)$ with $H^{2}=-\Lambda / 3$.

The other point to be noted here is that the speed of acoustic or sound waves $c_{s}$ is provided by Newton's formula $c_{s}^{2}=\partial p / \partial \rho$ which is real and less than the speed of light if $0 \leq \omega \leq 1$. Thus if $\omega=1$ i.e. $p=0$, we talk of pressure free matter or "dust"; if $\omega=1 / 3$ i.e. $p=\rho / 3$, we talk of "radiation" and if $\omega=1$ i.e. $p=\rho$ we talk of "stiff matter". Hence, acoustic waves in a radiation fluid travel at a speed of $1 / \sqrt{3}$ and this is the analogue for a gas of photons of "second sound" in a gas of phonons. BOOMERANG has observed the acoustic peaks in CMB. The dominant energy condition, though, allows both positive and negative pressure as long as $|p| \leq \rho$. The famous example of a single real scalar field $\phi$ with a potential $V(\phi)$ exibits this property when the field depends only on time. If the kinetic energy dominates we have stiff matter with maximum allowed positive pressure. But if the potential energy dominates, we get maximum negative pressure $p=-\rho$.

It is worth pointing out that the FRW ansatz necessarily implies an initial singularity called the Big Bang (scale factor vanishes at $t=0$, from which the universe starts expanding. For example, if $\rho+3 p>0$, from Raychaudhury equation we note that the acceleration of the universe, measured by $\ddot{a}$, is negative. For $k=-1,0$ Friedmann's equation says that for positive energy density, the universe naturally expands forever. whereas for $k=1, a$ will reach a value for which the curvature term compensates the energy density term yielding $\dot{a}=0$ after which $a$ decreases and the universe re-collapses, the Big Crunch.The total duration for light to propagate from the Big Bang to the Big Crunch can be found to be $2 \pi /|3 \omega+1|$. However, one should not be confused that a closed universe always re-collapses since Raychaudhury equation, which is independent of $k$, clearly states that for $\rho+3 p<0$ the universe will always accelerate. If the universe expands for ever and:

$$
r_{+}(t)=a(t) \int_{t}^{\infty} \frac{d t^{\prime}}{a\left(t^{\prime}\right)}<\infty
$$

there will be a future event horizon. de Sitter space time is an example of such a cosmological space time. Similarly if the scale factor vanishes at $t=0$, a Big Bang singularity, and

$$
r_{-}(t)=a(t) \int_{0}^{t} \frac{d t^{\prime}}{a\left(t^{\prime}\right)}<\infty
$$


then there will be a past horizon or particle horizon. If the scale factor goes to zero as $t^{n}$ near $t=0$, then there will be a particle horizon if $n<1$. For a $k=0$ universe one can show from the Einstein equation that $n=2 / 3$. Infact, since it is a good approximation to set $k=0$ always near a Big Bang because the term $k / a^{2}$ in Friedmann's equation is negligible compared with the other terms, we will have a particle horizon for the universes, no matter what the value of $k$ is. This puts the seeds for what is known as the horizon problem. The problem is the isotropy of the universe reflected by the CMB. Assuming the standard expansion of the universe we receive the same information from points in the space that do not appear to be in causal contact ( because of the particle horizon ) with each other. The actual puzzle is why the radiation is so uniform. This is one of the major problems of the standard cosmological model. For other problems associated with this model and also its successes, the reader should consult any text book on cosmology. All such problems strongly motivate us into possible ways of modifying the standard model of cosmology.

\subsection{Inflation}

The inflationary universe is a proposal, put forth about twentyfive years ago, to remedy the horizon problem and few others, not discussed here. The proposal postulated a rapid growth of the scale factor i.e. in the early universe there was a short time (inflationary period) when the universe expanded very fast. If the inflationary period is long enough it would explain why some regions could be in causal contact with each other, solving the horizon problem. It would also solve the flatness and other problems. We can define inflation to be any epoch where $\ddot{a}>0$, an accelerated expansion. We can rewrite this conditon in other ways as well:

$$
\ddot{a}>0 \Leftrightarrow \frac{d}{d t}\left(H^{-1} / a\right)<0 \Leftrightarrow p<-\rho / 3 .
$$

The middle definition has the most direct geometrical interpretation and it says that the Hubble length, as measured in comoving coordinates, decreases during inflation. At any other time, the comoving Hubble length increases. This is the key property of inflation; although typically the expansion of the universe is very rapid, the important characteristic scale of the universe is actually becoming smaller. when measured relative to that expansion. We should also bear in mind that the success of the standard model rely on the universe undergoing a conventional (non-inflationary) evolution and hence we cannot permit this inflationary period to continue for ever. On the contrary it must come to an end early enough so that the success of the standard model is not threatened. Thus inflation can be viewed as a phenomena of the very early universe. Note that inflation is not a replacement for the standard model, rather an improvement of the standard model during early evolution of the universe.

The simplest way to realize inflation, according to the third definition and our earlier discussion, is having a scalar field (called the inflaton in this context) $\phi$ with a potential $V(\phi)$ that provides an effective cosmological constant so that the scale factor increases exponentially. The equation of motion for the scalar field in the FRW metric is

$$
\ddot{\phi}+3 H \dot{\phi}+V^{\prime}(\phi)=0
$$

where prime denotes the derivative with respect to the scalar field. The energy density appearing in the Friedmann equation takes the form $\rho=\frac{1}{2} \dot{\phi}^{2}+V(\phi)$. Note that since anyway inflation 
will flatten the universe, we can ignore the curvature term in Friedmann equation. Also, inflation can occur if the evolution of the field is sufficiently gradual that the potential energy dominates the kinetic energy and the second derivative of the field is small enough to allow this evolution to be maintained for a sufficient period. Thus, we want $\dot{\phi}^{2} \ll V(\phi)$ and $|\ddot{\phi}| \ll|3 H \dot{\phi}|,\left|V^{\prime}\right|$. These requirements can be summarized as the following "slow-roll conditions" :

$$
\varepsilon \equiv \frac{M_{p l}^{2}}{2}\left(\frac{V^{\prime}}{V}\right)^{2} \ll 1 \eta \equiv M_{p l}^{2} \frac{V^{\prime \prime}}{V} \ll 1
$$

While the first condition implies the flatness of the potential, the second condition implies the slow rolling of the field and hence long enough period of inflation. The amount of inflation is measured by the number of e-foldings defined as

$$
N(t) \equiv \int_{t_{\text {init }}}^{t_{\text {end }}} H(t) d t=\frac{1}{M_{p l}^{2}} \int_{\phi_{\text {end }}}^{\phi_{\text {init }}} \frac{V}{V^{\prime}} d \phi
$$

To solve the horizon problem one requires $N>60$. To achieve this, one needs a careful choice of the potential function for the inflaton field and various potentials have been proposed for this purpose [1]. We should emphasize here that there is no compelling reason for the choice of any of these potentials.

A second scenario, proposed by Linde [2], called hybrid inflation, separates the inflaton from the ending of inflation. Thus there are at least two scalar fields involved in this proposal. While the inflaton has a flat potential and satisfies the slow roll conditions, the second field whose mass depends on the inflaton field in such a way that during and before the inflation its squared mass is positive but after inflation the squared mass becomes negative. Thus the second field becomes tachyonic and an instabilty appears in that direction i.e. the stationary point for this field becomes a maximum instead of a minimum and the field rolls fast towards the true vacuum which causes the inflation to end. We will see that both these scenarios can occur in the context of D-brane cosmology.

Before we end this section it is useful to mention some of the constraints on the model building of inflation imposed from experimental observations. Quantum fluctuations of the scalar field contributes to the fluctuations in energy density which indirectly accounts for the large scale structure formation. The observations imply a scale-invariant, Gaussian and adiabatic fluctuation in the temperature observed at COBE. Any scale, including the perturbations, increases substantially during inflation whereas the Hubble scale remains essentially same. Thus the scale leaves the horizon $\left(\sim H^{-1}\right)$ and the fluctations are frozen. But after the inflation, since Hubble scale increases at a faster rate the scales reenter the horizon. The amplitude of the density perturbation, when it reenters the horizon, as observed by CMB is given by

$$
\delta_{H}=\frac{2}{5} P_{R}^{1 / 2}=\frac{1}{5 \pi \sqrt{3}} \frac{V^{3 / 2}}{M_{p l}^{3} V^{\prime}}=1.91 \times 10^{-5},
$$

where $P_{R}$ is the power spectrum computed in terms of two-point correlators of the perturbations and the value of $\delta_{H}$ is taken from the COBE results. One can define an effective spectral index $n(k)$, to study the scale dependence of the spectrum, given by, $n(k)-1 \equiv \frac{d \ln P_{R}}{d \ln k}$. This is same as 
the assumed power law behaviour of the spectral index $P_{R} \propto k^{n-1}$ over an interval of $k$ when $n(k)$ is constant. $n(k)$ and its derivatives can be expressed in terms of slow-roll parameters as follows

$$
n-1 \simeq 2 \eta-6 \varepsilon, \frac{d n}{d \ln k} \simeq 24 \varepsilon^{2}-16 \varepsilon \eta+2 \xi^{2},
$$

where $\xi^{2} \equiv M_{p l}^{2} \frac{V^{\prime} V^{\prime \prime}}{V^{2}}$ i.e. for slow-roll the spectrum is almost scale invariant. Similarly the gravitational spectral index is found to be $n_{\text {grav }}=-2 \varepsilon$. One has to keep in mind these constraints when a potential coming from a physical context is being modeled for inflation.

\section{String Cosmology}

There has been various efforts from mid-1980s to understand cosmological implications of string theory, see [3], for a review. It is not possible even to outline the underlying ideas behind these efforts. Broadly speaking a class of models dealt with obtaining time dependent solutions of Einstein's equations obtained from low-energy effective action of string theory in presence of dilaton and with/without the two-form gauge field. There were some other interesting issues discovered around the same time. I will summarise below two such developments.

\subsection{Brandenberger-Vafa scenario}

This scenario exploited a stringy symmetry called T-duality. In the context of the closed bosonic and the heterotic string theory compactified on a circle of radius $R$, this symmetry states that the partition functions and mass spectrum remain invariant under $R \rightarrow \frac{1}{R}$. In the units where the string tension is half, the mass formula is given as

$$
M^{2}=m^{2} R^{2}+\frac{n^{2}}{4 R^{2}}+N_{L}+N_{R}-2,
$$

where the integers $m$ and $n$ correspond to the winding number and momentum in the circle respectively and $N_{L, R}$ are the left and right oscillator numbers. From this equation we see that the mass formula is invariant under the simultaneous exchange $R \leftrightarrow 1 / 2 R$ and winding and momenta $m \leftrightarrow n$. Brandenbergerand Vafa [4] pointed out that the concept of distance has different interpretation in the two dual regimes. For large $R$, the position coordinate is conjugate to the usual momentum $n / R$; but for distances smaller than the self-dual radius $(R=1 / \sqrt{2})$, one has to use the dual coordinate which is conjugate to the winding $m R$. Thus it does not make sense to talk about distances smaller than the string scale since they will be equivalent to large distances. Based on this observation, they conjectured that if the universe is considered to be product of circles, this could be the way to avoid the initial singularity. Further, they also had an interesting observation in support of a dynamical explanation for the reason why the universe looks to be four dimensional. This observation is based on the intution that the universe began with all the spatial dimensions being string size and the presence of winding modes preventing the expansion in the corresponding directions. But the winding modes can annihilate the anti-winding modes. In ten dimensions, a winding string need not meet the anti-winding string since their worldsheets can have many different trajectories. But these worldsheets can overlap in a four dimensional hypersurface in which the winding and the anti-winding strings annihilate each other to allow the expansion of the three 
spatial dimensions. Thus the punch line is that the winding strings prevent expansion in six spatial dimensions. However the question that remains unanswered is that what makes the universe to expand in this set up?

\subsection{Pre Big-Bang Inflation}

The equations of motion derived from the low energy string effective action for a FRW type background and the dilaton field $\phi$ is found to be invariant under the scale-factor duality symmetry i.e. for any solution $\{a(t), \phi(t)\}$, there is also a solution with $a^{\prime} \rightarrow a^{-1}, \phi^{\prime} \rightarrow \phi-2 \sum_{i} \ln a_{i}$, which is infact the T-duality of this background. Note that the scale factor duality relates the expanding and the contracting universes i.e. solutions with decreasing curvature are mapped to those with increasing curvature.It was also observed that the equations of motion were not only invariant under T-duality but also under $a(t) \leftrightarrow a(-t)$. Though, under $t \rightarrow-{ }^{\prime}$, we get $H(t) \rightarrow-H(-t)$; when combined with the duality symmetry, we do have $H(t)=H(-t)$. Thus it possible to have a period of inflation before $t=0$. This led to the Pre Big-Bang inflationary scenario [5], in which the universe starts out as flat empty space, begins to contract with increasing curvature, until reaching a stringy state of maximum curvature and then expands with decreasing curvature and leads to the standard cosmological evolution. This scenario, possibly, can help to provide the initial conditions for standard evolution of the universe. However, it is essential to understand whether the symmetry, $a(t) \rightarrow a(-t)$ is preserved under the stabilization of the dilaton field by non-perturbative effects. Another issue, namely the graceful exit in this scenario, is studied in [6] by including both the dilaton and the axion field in the effective action. It was found that the conservation of axionic charge leads to a potential function involving the dilaton. The Wheeler-de Witt equation was solved exactly and appropriate wave function satisfying the required boundary conditions to solve the graceful exit problem were obtained.

\section{Cosmology with Branes}

Cosmology has taken a different route in the context of string theory by taking the advantage of the existence of various types of solitonic objects called branes or D-branes, which are either supersymmetric (BPS -branes) or non-supersymmetric (non-BPS-branes). The imagination, in the context of brane cosmology, is that we are living on one of the brane. In this spirit, the reason that the extra spatial dimensions, predicted by string theory, are invisible to us is not because of the fact that they are so tiny to be probed by low-energy excitation, but because we are confined to a three-dimensional brane embedded in a higher-dimensional space. One can construct field theories like the entire Standard Model of particle physics by suitable arranging a stack of branes (although it has not be accomplished yet). However, it does not mean that one can do away with the necessity of compactification, since we donot know how gravitational force can be confined to a brane. Infact, it does not. Neverthless, cosmology on branes or braneworld cosmolgy, as it is known, has met reasonable success starting with the work of [7]. These interesting developments are not addressed in this note since they are already discussed in this workshop [8], including the braneantibrane inflation with special emphasis on fine tuning for a flat potential, in the framework of [9] for stabilization of the moduli fields. Instead, I will discuss another aspect of brane cosmology involving non-BPS branes. 


\subsection{Tachyon Cosmology}

The existence of tachyons is an intrinsic part of string theory, both open and closed. In closed string theory, they are projected out by the GSO mechanism though this projection has not been checked beyond two loops in the perturbation theory. In open string theory tachyons always make their presence felt signalling an instability of the perturbative vacuum. For example, a tachyonic excitation is always present in the spectrum of the open string, either stretched between a brane and an antibrane (both are BPS) or stuck on a non-BPS brane. The dynamics of such a tachyon field has been greatly understood by the pioneering work of Sen, see for a review [10]. The tachyon field $T(x)$ will always roll toward the true vacuum, with zero energy density, from the unstable perturbative vacuum. Most of the aspects of this process can be captured by simple effective field theory models for the tachyon field. For example, in the case of the superstring, the boundary string field theory, one obtains the following effective Lagrangian,

$$
\mathscr{L}_{b s f t}=-\sqrt{-g} e^{-T^{2}}\left(1+\partial_{\mu} T \partial^{\mu} T\right)
$$

where $T$ stands for the modulus of the complex tachyon field for this case. The cosmological implication for the open-string tachyon field was studied, for the first time, in a brane-world scenario [11] by coupling the above effective theory to gravity. It was found that in a single brane scenario, the slow-roll conditions, described in the previous section, are not satisfied. However, in the framework of assisted inflation with about ten such branes, one can have a viable model for inflation and all the observational constraints, as mentioned in the previous section, could be satisfied.

The above effective Lagrangian was obtained with some approximations in a complicated analysis of string field theory. By invoking purely to T-duality symmetry arguments and some scattering amplitude analysis, a Dirac-Born-Infeld type of action was proposed in [12], which has been found to capture most of the tachyon condensation process. This form of the effective Lagrangian is given by

$$
\mathscr{L}_{d b i}=-\sqrt{-g} V(T) \sqrt{1+g^{\mu v} \partial_{\mu} T \partial_{v} T}
$$

The potential $V(T)$ ( $T$ is real scalar for a non-BPS brane) is non-negative and has a unique local maximum, with height being equal to the tension of the brane, at $T=0$ and local minima at $T=\infty$ where the potential vanishes. Small fluctuations around the false vacuum at $T=0$ have negative mass squared and hence it is unstable. From the equation of motion for the tachyon field one can deduce that the tachyon is not a tachyon and no super-luminal propagation is ever possible contrary to popular prejudice [13]. The rolling of the tachyon to its true vacuum, which is actually the closed string vacuum and the process is the decay process of the unstable brane. Note that the energy density is still localized. This is the essence of tachyon condensation. Cosmological relevance of this tachyon field has been studied [14] by coupling this to gravity for FRW background and using the tachyon potential derived from string theory; many authors have also studied this using phenomenological chosen potentials. For a flat universe, it has been found that accelerated expansion takes place for $\dot{T}^{2}<2 / 3$. The slow-roll parameters have been derived for this non-canonical scalar field action and it has been observed that the $\eta \ll 1$ condition is not satisfied within the framework of effective field theory with weak gravity. Thus the number of e-foldings turn out to be around 20 which is much less than the desired number, $N>60$. However, 
considering a non-minimal coupling of the tachyon field with gravity, the coupling form could be motivated from string theory, and invoking to a warped compactification, instead of dimensional reduction, not only the slow-roll condition could be satisfied but also other observational constraints, including required number of e-foldings, were under control [15].

In the context of tachyon driven cosmology, it was observed in [16], by using open-closed string duality, that a time reversal invariant bounce solution with positive spatial curvature can be obtained. Moreover, in the absence of a bulk cosmological constant, the universe always begins with a big bang and ends in a big crunch.

It is worth pointing out that the above DBI field theory coupled to gravity has been studied in the framework of canonical quantization in [17] to study the possibility of using the tachyon field as the definition of time in quantum cosmology. Inspite of some success, tachyon cosmology has been subjected to some criticism like lack of a mechanism for reheating and over-aboundance problem. However, it has been argued in [18] that this problem can be naturally solved if almost all of the tachyon energy is drained (via coupling of matter fields) to reheat the universe, while the rest of the energy goes to lower dimensional BPS D-branes produced during the tachyon rolling at the end of inflation.

In the context of inflation from brane-antibrane annihilation, the open-string tachyon plays a crucial role for the universe to exit from the inflationary epoch. This is shown in [19] by constructing a hybrid inflationary model of the scalar field corresponding to the separation of the brane and the antibrane and the tachyon field. The tachyon field has no role till the brane-antibrane separation becomes in the order of the string length. The results, however, were found to be sensible to initial configuration and the geometry of the internal manifold.

\subsection{Cosmology from Geometric tachyon}

In [20], the time dependent motion of a probe D-brane in a coincident NS5-brane back ground was studied and it was observed that there is a map between the radial field living on the D-brane world volume and the rolling tachyon of the non-BPS brane and hence the motion of the probe D-brane in the background geometry of NS5-branes can be described by the DBI type Lagrangian discussed earlier. Furthermore, it was also observed [21] that by compactifying one of the transverse directions to the source brane, it is possible to obtain a tachyon potential. The result suggests that the tachyon could have a geometrical origin. The map between the the radion and the tachyon field and kink solution for the geometrical tachyon has been further studied in [22]. The cosmological relevance of this geometrical tachyon was explored in [23] where a D3-brane was used as the probe brane. It was found that cosmological observables in this model, including the spectra of scalar and tensor perturbations are compatible with the recent observations of CMB. However, the number of sourse NS5- branes needed was found to be too high. The S-dual version of this model, i.e. the motion of a D3-brane in the background of a stack of D5-branes was considered in [24]. In this set up, inflation is realized by the potential of the geometric tachyon field, consistent with CMB data and much less number of source D5-branes. When the separation between probe D3brane and stack of source D5-branes becomes within the string length scale (after the inflation is over), a complex tachyon field develops in the world volume of the probe brane. Thus the late time dynamics is governed by this tachyon field, described by an action with a canonical kinetic term and a potential. Thus reheating can be successfully realized with spontaneous symmetry breaking. 
I end this note by mentioning that topics like cosmology with closed string tachyon condensation, brane gas model and possibly few others could not be discussed in allowed time and I offer my apology to colleagues who have contributed to such topics. I also thank my collaborators for sharing their insight with me during our investigations.

\section{References}

[1] A. R. Liddle and D. H. Lyth, Cosmological Inflation and Large-Scale Structure (Cambridge University Press), 2000.

[2] A. Linde, Phys. Rev. D 49748 (1994).

[3] J. E. Lidsey, D. Wands and E. J. Copeland, Phys. Rept. 337343 (2000).

[4] R. Brandenberger and C. Vafa, Nucl. Phys. B 316391 (1989).

[5] M. Gasperini and G. Veneziano, Astropart. Phys. 1317 (1993); see also the web page http://www.to.infn.it/gasperin/.

[6] J. Maharana, S. Mukherji and S. Panda, Mod. Phys. Lett. A 12447 (1997).

[7] N. Arkani-Hamed, S. Dimopoulos and G. R. Dvali, Phys. Rev. D 59086004 (1999).

[8] J. M. Cline, Leture notes on Braneworld Cosmology and lecture note on Fine-Tuning in Brane-antibrane Inflation, delivered in this workshop and references therein.

[9] S. Kachru, R. Kallosh, A. Linde, J. M. Maldacena, L. McAllister and S. P. Trivedi, JCAP 0310013 (2003).

[10] A. Sen, Int. J. Mod. Phys. A 205513 (2005).

[11] A. Mazumdar, S. Panda and A. Perez-Lorenzana, Nucl. Phys. B 614 (2001).

[12] E. A. Bergshoeff, M. de. Roo, T. C. deWit, E. Eyras and S. Panda, JHEP 0005009 (2000); M. R. Garousi, Nucl. Phys. B 584 (2000).

[13] G. W. Gibbons, arXiv: hep-th/0301117

[14] M. Fairbairn and M. H. Tytgat, Phys. Lett. B 5461 (2002); D. Choudhury, D. Ghoshal, D. P. Jatkar and S. Panda, Phys. Lett. B 544231 (2002)

[15] A. Deshamukhya, P. Chingangbam and S. Panda, JHEP 02052 (2005).

[16] A. Sen, arXiv: hep-th/0312153.

[17] A. Sen, Int. J. Mod. Phys. A 184869 (2003).

[18] G. Shiu, S.-H. Henry Tye and I. Wasserman, arXiv: hep-th/0207119.

[19] D. Choudhury, D. Ghoshal, D. P. Jatkar and S. Panda, JCAP 07009 (2003).

[20] D. Kutasov, arXiv: hep-th/0405058.

[21] D. Kutasov, arXiv: hep-th/0408073.

[22] S. T. Thomas and J. Ward, arXiv: hep-th/0411130, hep-th/0502228.

[23] S. Panda, M. Sami and S. Tsujikawa, Phys. Red. D 73023515 (2006).

[24] S. Panda, M. Sami, S. Tsujikawa and J. Ward Phys. Red. D 73083512 (2006). 